\title{
Análisis del comportamiento de las propiedades geométricas de los contornos de huecos en nubes de puntos
}

\section{Analysis of geometrical properties behavior of holes contours in cloud of points}

\author{
Germán Sánchez T. ${ }^{1}$, Esmeide Leal ${ }^{2}$, Nallig Leal ${ }^{3}$ \\ ${ }^{1}$ PhD en Ingeniería. Profesor asistente, Universidad del Magdalena, Santa Marta. Grupo de I+D \\ en nuevas tecnologías de la información y la comunicación GIDTIC. Email: gsanchez@unimagdalena.edu.co. \\ ${ }^{2} \mathrm{MSc}$ en Ingeniería de sistemas. Profesor titular, Universidad Autónoma del Caribe, Barranquilla. \\ Programa Análisis y Diseño de Sistemas y Computación. Email: esleal@uac.edu.co. \\ ${ }^{3}$ MSc en Ingeniería de sistemas. Profesor titular, Universidad Autónoma del Caribe, Barranquilla. \\ Programa de Ingeniería de Sistemas. Email: nleal@uac.edu.co.
}

Recibido 28/10/13, Aceptado 22/12/2013

\section{RESUMEN}

Este trabajo está relacionado con las anomalías representadas por discontinuidades o huecos presentes en nubes de puntos tridimensionales. Las auto-oclusiones y las propiedades ópticas del material del objeto sensado constituyen las fuentes principales de anomalías. El presente estudio fue realizado con el propósito de analizar similitudes o disimilitudes estadísticas de las propiedades geométricas de los contornos generados por estas dos fuentes. Las propiedades analizadas fueron la estimación de la curvatura y la torsión, para esto se clasificaron los diferentes contornos en dos grupos: los generados por propiedades ópticas del material del objeto y los generados por oclusión. Los resultados sugieren que estas fuentes generan contornos de discontinuidades estadísticamente similares, dificultando una discriminación basada en estas propiedades.

Palabras clave: Curvatura, Llenado de huecos, Oclusiones, Reconstrucción de superficies, Torsión.

\begin{abstract}
This work is related to anomalies characterized by discontinuities called holes on three-dimensional cloud of points. The self-occlusions and optical properties of scanned objects are the main source generating anomalies. The present study is making in order to analyze statistical similarities and dissimilarities of geometrical properties of contour generated by these sources. The analyzed properties were the curvature and torsion estimations, for this, each one of contours was classified into two groups: those generated by optical properties and those generated by occlusion. The results suggest those sources generate similar statistical holes, making it difficult to make a discrimination based on these properties.
\end{abstract}

Keywords: Curvature, Hole filling, Occlusions, Surface reconstruction, Torsion.

\section{INTRODUCCIÓN}

Inferir el valor de algún parámetro de un sistema desconocido a partir de un conjunto de medidas conocidas de dicho sistema es un problema clásico en ciencias denominado Problema Inverso. Recuperar la estructura tridimensional desde nubes de puntos tridimensionales es un ejemplo de un problema inverso en ingeniería, comúnmente llamado Reconstrucción de Superficies. La Reconstrucción de Superficies es un campo cuyo objetivo es determinar una superficie matemática $\omega$ que represente a una superficie real desconocida s, de tal forma que; mediante un conjunto de datos conocidos. El conjunto de los constituye el conjunto de observaciones y en este contexto son conocidos como nubes de puntos.

Las nubes de puntos como fuente de información para reconstruir superficies de objetos reales poseen una popularidad que inició hace algo más de dos décadas con el trabajo de Hoppe et al. [1]. Su uso ha seguido creciendo debido al alto desarrollo de las tecnologías láser que brindan significativas mejoras en los niveles de precisión y flexibilidad para la adquisición de datos de rango [2]. Otro aspecto que ha permitido la generalización del uso de este tipo de imá- 
genes es que éstas representan de forma explícita la geometría de la superficie de la escena [3] (ver figura 1). Diferentes etapas componen el proceso de reconstrucción de superficies; la adquisición, el registro, la integración y el ajuste. El interés de la problemática abordada se centra en la etapa de integración, cuya dificultad radica en que se inicia con un conjunto de datos en el espacio tridimensional cuya conectividad y relación aún no está bien definida y su importancia consiste en disminuir el efecto de estas anomalías en los procesos de representación final.

Figura 1. Visualización de la nube de puntos del objeto Bunny

Figure 1. Visualization of point cloud of Bunny object

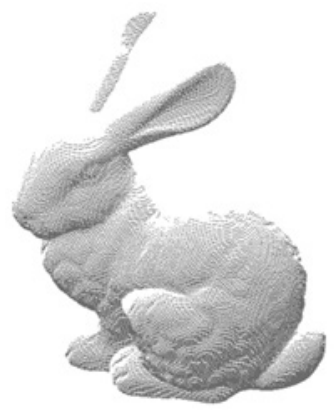

La idea tras la corrección de este tipo de anomalías consiste en reproducir el segmento de superficie faltante que se incorporará a la malla inicial con el fin de reparar la anomalía. En este sentido, diferentes técnicas han sido propuestas en [4 - 10]. En este grupo de técnicas basadas en geometría se encuentran dos tendencias generales. La reparación basada en triángulos y la reparación basada en voxels.

Las técnicas basadas en triángulos obtienen mediante triangulación una aproximación lineal del hueco y refinan esta triangulación de acuerdo con la geometría en la cual el hueco está inmerso. Algunos ejemplos de estas técnicas son [11-12], que aunque generan soluciones visuales adecuadas, sin embargo, son costosos en tiempo computacional y están limitados al tamaño del hueco. Por otra parte, los procedimientos basados en voxels generan una representación volumétrica de la superficie inicial compuesta de un conjunto de unidades de volumen llamadas voxels, $[7,13,14]$, presentaron algoritmos para reparar discontinuidades basados en voxels.

En general, los trabajos en el estado del arte realizan correcciones de los modelos 3D sin ninguna consideración geométrica diferente de la discontinuidad del modelo, lo que los limita al tratamiento de objetos continuos y dificulta el tratamiento de modelos que presenten discontinuidades reales, debido a que no proponen esquemas de clasificación de discontinuidades que les permita asumir la fuente de la anomalía.
Este trabajo está orientado a analizar las características de las anomalías formadas por fenómenos ópticos causados por las propiedades físicas del material del objeto y las características causadas por oclusiones, con el objetivo de establecer diferencias que permitan discriminar la fuente que genera la anomalía. Dado que los contornos de los huecos forman curvas tridimensionales, éstas pueden ser caracterizadas por las estimaciones de curvatura y torsión. De esta forma, se conformó un conjunto de contornos que fueron clasificados de acuerdo a la fuente en dos grupos de acuerdo a la fuente. Para cada grupo, se estimó una aproximación por pedazos de la curva que describe el contorno de cada hueco. Esta aproximación fue realizada mediante una secuencia de curvas de Bézier, lo que permite obtener una medida de las propiedades geométricas, que posteriormente se analizaron estadísticamente.

El documento se estructuró con una descripción metodológica, seguida de la presentación y descripción de los resultados obtenidos. Finalmente, se muestran las principales conclusiones obtenidas.

\section{METODOLOGÍA}

Las anomalías presentes en nubes de puntos provienen de una amplia variedad de fuentes que pueden encontrarse en diferentes etapas del proceso. Las principales fuentes de generación de discontinuidades están ubicadas en la etapa inicial de adquisición y ocasionan que en algunas regiones sea inviable, muy costoso o impráctico adquirir la información, generando huecos que no están presentes en el modelo real, éstos huecos constituyen un conjunto de micro-agujeros de diversas formas. A pesar de que los microagujeros representan el caso más frecuente, el tamaño, así como su forma, constituyen características altamente variables, por lo que para este estudio, la forma y el tamaño no se consideraron variables relevantes. Así, las oclusiones y las propiedades ópticas son las fuentes generadoras de anomalías más importantes del proceso.

Las oclusiones impiden que segmentos de la superficie real sean adquiridos generando un conjunto de datos incompletos afectado por discontinuidades artificiales. Diversos escenarios pueden involucrar oclusiones, la mayoría de éstas, sólo afectan las vistas parciales en las cuales un reposicionamiento de la configuración del montaje de adquisición es suficiente para adquirir dicha información. Sin embargo, en algunas ocasiones partes del objeto ocultan segmentos que no son visibles desde ningún punto de vista del sensor y son llamadas auto-oclusiones. En [15], se describe dos tipos de oclusiones, oclusión láser y oclusión de cámara (ver figura 2). La oclusión láser ocurre cuando el láser es incapaz de iluminar un punto del objeto visible por la cámara. Por otra parte, la oclusión de cámara ocurre cuando la cámara no puede adquirir un punto iluminado por el láser. 
Figura 2. Tipos de oclusión, a) oclusión laser, b) oclusión de cámara [15]

Figure 2. Types of occlusion, a) laser occlusion, b) camera occlusion [15]

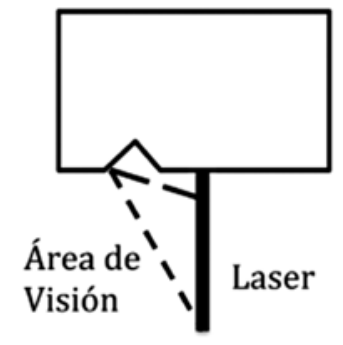

a)

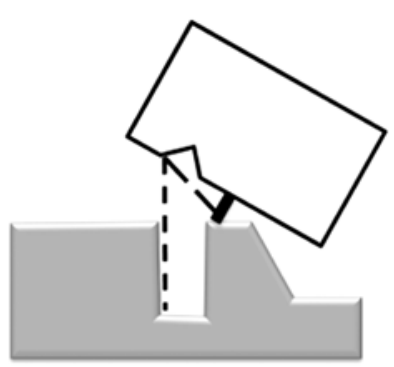

b)

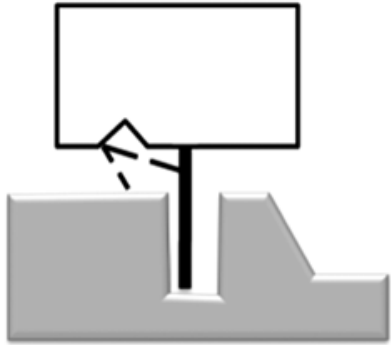

c)
En general, las propiedades ópticas de un material están determinadas por la forma en que la luz interactúa con éste. La reflexión puede producirse en una superficie lisa y completamente pulida o sobre una superficie irregular. Sin embargo, los objetos no presentan superficies que son especulares ni difusoras por completo, así, el resultado es una reflexión mixta que tiene componente especular representado en el brillo y componentes difusas que permiten apreciar características como su color.

\subsection{Selección de objetos y generación de imágenes}

La selección del conjunto de objetos fue realizada teniendo en cuenta que sus materiales produjeran las diferentes fuentes de generación de anomalías. En la figura 3, se muestran algunos objetos del conjunto de prueba y una de las vistas del proceso de adquisición. Las imágenes obtenidas corresponden a un proceso de adquisición bajo las mismas condiciones de escala y calibración.

Figura 3. Ejemplos del conjunto de objetos con materiales brillantes (a), y del conjunto de objetos opacos (b), así como sus una de las vistas adquiridas (c y d)

Figure 3. Examples of brilliant (a) and opaque (b) object set and one partial view (c and d) from acquisition process
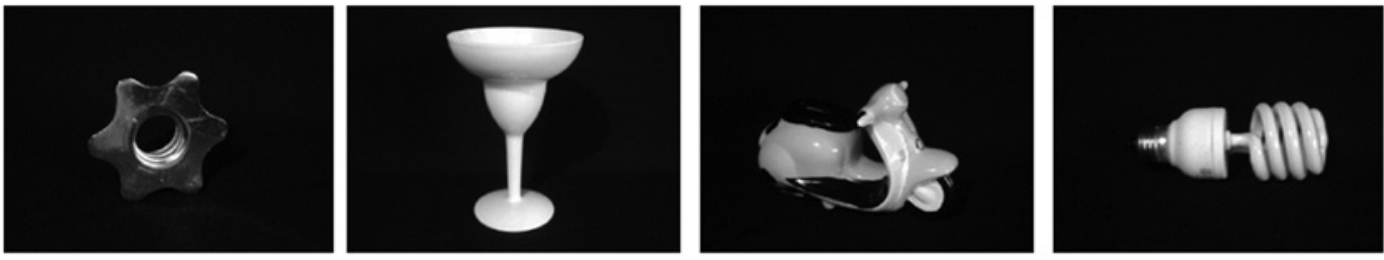

a)
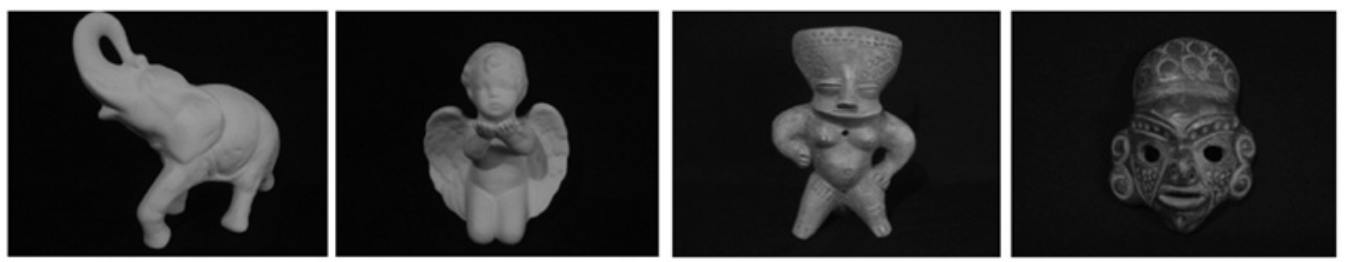

b)
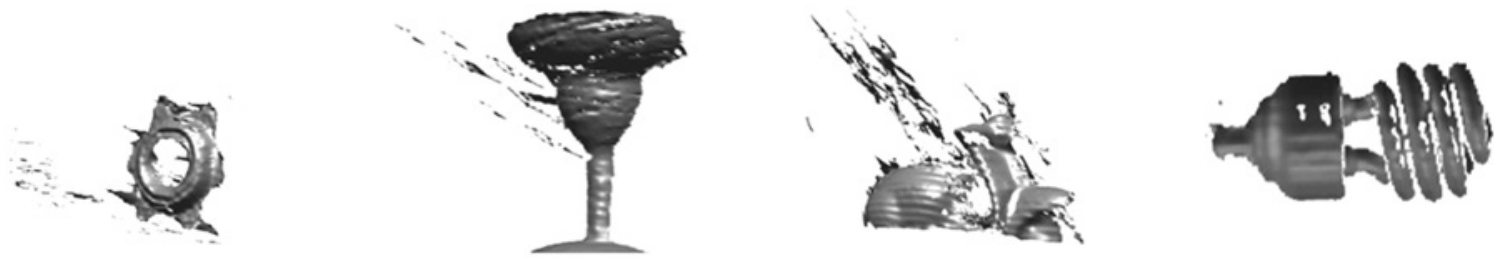

c) 

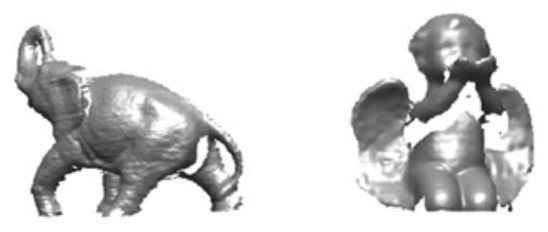

d)

\subsection{Extracción de contornos y estimación de características geométricas}

Para obtener cada contorno se estimó una representación triangular de las superficies adquiridas. Diferentes trabajos describen diversas técnicas para la identificación de discontinuidades sobre mallas triangulares [1-16]. El procedimiento más sencillo y funcional con gran facilidad de implementación lo constituye el propuesto por [17]. En este trabajo, una discontinuidad en la superficie es definida como una sucesión de aristas límite. Una arista límite se define como un borde de un triángulo que no es compartido por ningún otro.

Una vez obtenidos los contornos el siguiente paso consiste en estimar una aproximación continua del contorno. Esto es necesario debido a que el contorno está descrito por una secuencia cerrada de aristas y vértices, por lo tanto, éstas se aproximan mediante curvas de Bézier, teniendo en cuenta que la función que describa la curva debe ser diferenciable, hasta por lo menos en tercer grado. Una curva paramétrica de Bézier se define así:

$$
\begin{aligned}
& x(t)=\sum_{i=0}^{n} x_{i} B_{i, n}(t) \\
& y(t)=\sum_{i=0}^{n} y_{i} B_{i, n}(t) \\
& z(t)=\sum_{i=0}^{n} z_{i} B_{i, n}(t)
\end{aligned}
$$

En general

$$
\mathrm{Cb}(\mathrm{t})=\sum_{\mathrm{i}=0}^{\mathrm{n}} \mathrm{p}_{\mathrm{i}} \mathrm{B}_{\mathrm{i}, \mathrm{n}}(\mathrm{t})
$$

Donde, B son los polinomios de Berstein de tercer grado y se define así:
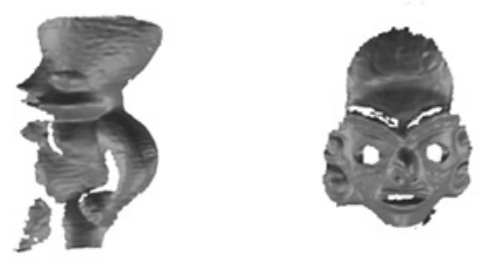

$$
\begin{aligned}
& \mathrm{B}_{0,3}=(1-\mathrm{x})^{3} \\
& \mathrm{~B}_{1,3}=3 \mathrm{x}(1-\mathrm{x})^{2} \\
& \mathrm{~B}_{2,3}=3 \mathrm{x}^{2}(1-\mathrm{x}) \\
& \mathrm{B}_{3,3}=\mathrm{x}^{3}
\end{aligned}
$$

Una vez obtenida la función, la curvatura y la torsión pueden estimarse así:

$$
\tau(s)=-\frac{\left(r^{\prime} \times r^{\prime \prime}\right) \cdot r^{\prime \prime \prime}}{\left\|r^{\prime} \times r^{\prime \prime}\right\|^{2}} \quad \mathrm{k}(s)=\frac{\left(r^{\prime} \times r^{\prime \prime}\right)}{\left\|r^{\prime}\right\|^{3}}
$$

\section{RESULTADOS}

A cada contorno extraído se le estimó un grupo de medidas de torsión y curvatura, de igual tamaño que el número de vértices que conforma el contorno. La figura 4, muestra el comportamiento de los valores obtenidos para cada grupo de contornos, tanto para la curvatura (figura 4a), como para la torsión (figura 4b). Los estadísticos que describen dicho comportamiento se muestran en la tabla 1.

En la figura 4, es posible notar que estos dos grupos de medidas tienden a comportarse de manera similar. Con una ligera diferenciación en los niveles de curvatura y torsión para el grupo de objetos brillantes que muestra valores superiores frente a los del grupo de objetos opacos. Por otra parte, el grupo de contornos opacos muestra un rango superior en relación con el rango de los contornos brillantes. Sin embargo, no es posible observar una diferenciación definitiva para afirmar que estas medidas se comportan de forma diferente de acuerdo con la naturaleza de la anomalía. La tabla 1, muestra promedios muy cercanos y desviaciones estándar similares para ambos grupos.

Debido a la dificultad de obtener un concepto determinante frente al comportamiento de las dos medidas obtenidas de los diferentes grupos analizados, se realizaron pruebas estadísticas para determinar si las diferencias encontradas son significativas para determinar si estos comportamientos son o no diferentes. 
Figura 4. Comportamiento del promedio de a) curvatura y b) la torsión para los conjuntos de contornos extraídos de objetos brillantes (línea punteada) y opacos (línea continua)

Figure 4. Average behavior of a) curvature y b) torsion of contour set from brilliant (dashed line) and opaque (continuous line) object set

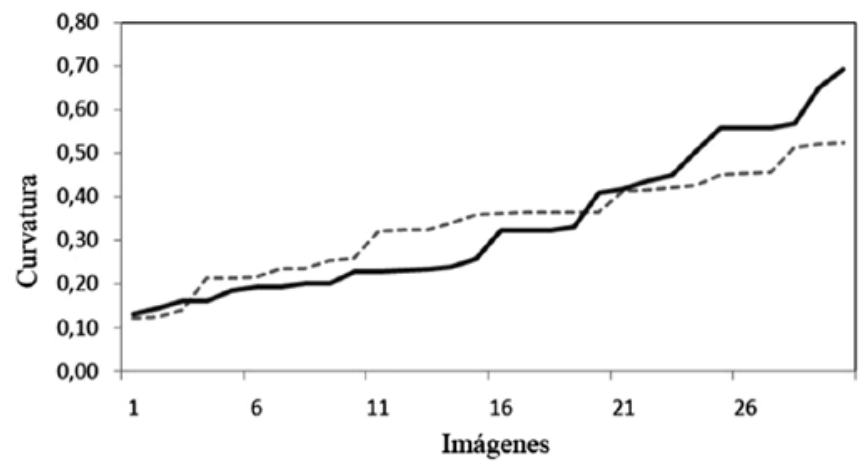

a)

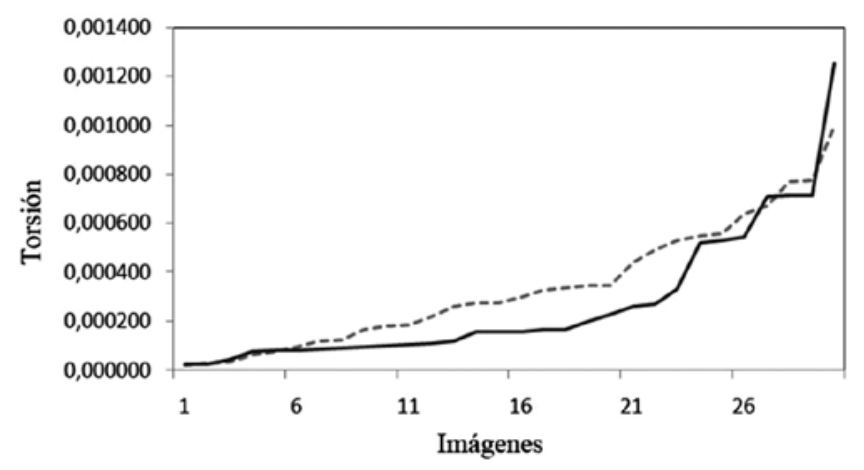

b)

Tabla 1. Estadísticas de datos para curvatura y torsión Table 1. Statistic measures from curvature and torsion data set

\begin{tabular}{|c|c|c|c|c|}
\hline & \multicolumn{2}{|c|}{ Curvatura } & \multicolumn{2}{c|}{ Torsión } \\
\hline & Brillantes & Opacos & Brillantes & Opacos \\
\hline Rango & 0,401 & 0,562 & 0,0009 & 0,00122 \\
\hline Promedio & 0,3366 & 0,3377 & 0,0003 & 0,00027 \\
\hline Desv. Estándar & 0,1144 & 0,1654 & 0,00025 & 0,00028 \\
\hline
\end{tabular}

\subsection{Prueba de hipótesis para las medias}

Es posible notar que las medias de 0,3365 y 0,3377 para la curvatura, y $3,41 \times 10^{-4}$ y $2,70 \times 10^{-4}$ para la torsión, son muy cercanas en cada grupo. Un factor determinante e inicial para realizar análisis estadísticos lo constituye la prueba de normalidad en los datos. Debido a las características de los datos, en relación con su tamaño, la prueba
Shapiro-Wilk resulta más adecuada [18]. Los resultados de la prueba de normalidad se describen en la tabla 2a y la tabla $2 b$, para los grupos de muestras de curvatura y torsión respectivamente. Las pruebas fueron realizadas con un nivel $\alpha=0,05$, asumiendo la normalidad de la distribución si el estadístico obtenido es no significativo aceptando la normalidad de los datos para $>0,05$. Los resultados muestran que a pesar de que la distribución de los datos de la muestra de curvaturas obtenidas en objetos brillantes, está distribuida de forma normal, el resto de las muestras no presentan ese comportamiento (ver tabla 2). Debido a la no normalidad de los datos, se aplicó una prueba no paramétrica para analizar la similitud entre las medias de ambos pares de grupos. Se aplicó la prueba de Mann-Whitney, apropiada para cuando la asunción de normalidad en la distribución de los datos no es posible. Las hipótesis utilizadas para la comparación de los grupos fueron: la hipótesis nula : Las dos muestras provienen de idénticas poblaciones y la hipótesis alternativa : Las dos muestras provienen de poblaciones diferentes. A un nivel de significancia de $\alpha=0,05$ y una regla de decisión tal que: $\mathrm{si}<0,05$ se rechaza la hipótesis nula. Los resultados obtenidos se muestran en la tabla 3. Los estadísticos encontrados para el grupo de curvaturas y de torsión corresponden a 0,605 y 0,117 . De tal forma que para ambos casos se acepta la hipótesis nula.

Tabla 2. Resultados de las Pruebas de normalidad para a) curvatura y b) torsión

Table 2. Results of a) curvature and b) Torsion normality tests

\begin{tabular}{|l|c|c|c|c|c|c|}
\hline \multirow{2}{*}{} & \multicolumn{3}{|c|}{ Kolmogorov-Smirnov } & \multicolumn{3}{c|}{ Shapiro- Wilk } \\
\cline { 2 - 7 } & Statistic & $\mathrm{df}$ & Sig. & Statistic & $\mathrm{df}$ & Sig. \\
\hline C_Brillantes & 0,114 & 30 & 0,200 & 0,957 & 30 & 0,264 \\
C_Opacos & 0,187 & 30 & 0,009 & 0,901 & 30 & 0,009 \\
\hline
\end{tabular}

a)

\begin{tabular}{|c|c|c|c|c|c|c|}
\hline & \multicolumn{3}{|c|}{ Kolmogorov-Smirnov } & \multicolumn{3}{c|}{ Shapiro- Wilk } \\
\cline { 2 - 7 } & Statistic & df & Sig. & Statistic & df & Sig. \\
\hline T_Brillantes & 0,240 & 30 & 0,000 & 0,564 & 30 & 0,000 \\
T_Opacos & 0,375 & 30 & 0,000 & 0,311 & 30 & 0,000 \\
\hline
\end{tabular}

Tabla 3. Resultados de la prueba de homogeneidad de varianzas

Table 3. Results of the homogeneity of variances test

\begin{tabular}{|c|c|c|}
\hline \multirow{2}{*}{} & \multicolumn{2}{|c|}{ Levene's Test for Equality of Variances } \\
\cline { 2 - 3 } & $\mathrm{F}$ & Sig. \\
\hline Curvaturas & 6,083 & 0,17 \\
\hline Torsión & 0,29 & 0,866 \\
\hline
\end{tabular}




\section{CONCLUSIONES}

- Lo resultados obtenidos del análisis de las curvas que representan los contornos de anomalías causadas por fenómenos ópticos y por oclusión, sugieren que las diferencias encontradas pueden considerarse productos del azar.

- El comportamiento de los valores de curvatura y los valores de las torsiones no permite que éstas puedan ser consideradas como características discriminantes para determinar una fuente generadora de anomalías asociadas a ausencia de información, de forma similar al tamaño y la forma. Por lo tanto, en el diseño de un método para el tratamiento de correcciones se puede asumir un tratamiento similar para las anomalías con independencia de la fuente que la genera.

- Estos resultados evidencian la dificultad en la discriminación de la fuente tomando sólo la estimación de las características. Sin embargo, de acuerdo con los datos obtenidos, existe evidencia que sugiere que la distribución de los valores de las características medidas, en cada grupo es diferente. Es decir, aunque los promedios y los valores sean similares para ambas fuentes de anomalías, estos valores presentan diferencias en las distribuciones dentro del rango numérico. Este hecho permite asumir que es posible, la construcción de un clasificador más complejo, que uno que sólo toma en cuenta el valor numérico de las características geométricas.

- Como trabajo futuro, se propone el análisis de las regiones de superficie cercanas al contorno para determinar si existen perturbaciones introducidas por la fuente de generación de la discontinuidad, y si estas perturbaciones pueden caracterizarse con el objeto de diferenciar la fuente generadora.

\section{REFERENCIAS}

[1] Hoppe, H., DeRose. T., Duchamp T., McDonald J., and Stuetzle, W., Surface reconstruction from unorganized points. SIGGRAPH Comput. Graph. 26 (2), 71-78, 1994.

[2] Forster, F., A high-resolution and high accuracy real-time 3D sensor based on structured light. 3D Data Processing, Visualization, and Transmission, Symposium, Munich (June), pp. 208-215, 2006.

[3] Coleman, S., Scotney, B., and Suganthan, S. Feature extraction on range images - a new approach. In Robotics and Automation, IEEE International Conference, Roma, $1098-1103,2007$.

[4] Dorai, C., Wang, G., Jain, A. K., and Mercer, C. Registration and integration of multiple object views for $3 \mathrm{~d}$ model construction. IEEE Transactions on Pattern Analysis and Machine Intelligence, 20(1), 83-89, 1998.
[5] Davis, J., Marschner, S. R., Garr, M., and Levoy, M. Filling holes in complex surfaces using volumetric diffusion. Symposium on 3D Data Processing, Visualization, and Transmission, Padua, Italy, 428-438, 2002.

[6] Wang, J. and Oliveira, M. Filling holes on locally smooth surfaces reconstructed from point clouds. Image and Vision Computing, 25(1), 103-113, 2007.

[7] Podolak, J. and Rusinkiewicz, S. Atomic volumes for mesh completion. Proceedings of Eurographics symposium on Geometry processing, Switzerland, 33-41, 2005.

[8] Bischoff, S., Pavic, D., and Kobbelt, L. Automatic restoration of polygon models. ACM Trans. Graph., 24(4), 1332-1352, 2005.

[9] Guo, T., Li, J., Weng, J., and Zhuang, Y. Filling holes in meshes and recovering sharp edges. Systems, Man and Cybernetics, 6, 5036-5040, 2006.

[10] Branch, J., Prieto, F., and Boulanger, P. Automatic hole-filling of triangular meshes using local radial basis function. Symposium on 3D Data Processing, Visualization, and Transmission (3DPVT'06), Washington, DC, USA. IEEE Computer Society, 727-734, 2006.

[11] Liepa, P. Filling holes in meshes. Eurographics/ACM SIGGRAPH symposium on Geometry processing, Switzerland, 200-205, 2003.

[12] Zhao, W., Gao, S., and Lin, H. A robust hole-filling algorithm for triangular mesh. Vis. Comput., 23(12), 987-997, 2007.

[13] Ju, T. Robust repair of polygonal models. ACM Transactions on Graphics, 23(3), 888- 895, 2004.

[14] Curless, B. and Levoy, M. A volumetric method for building complex models from range images. Computer graphics and interactive techniques, New York, NY, USA. ACM Press, pp. 303-312, 1996.

[15] Banta, J., Wong, L. Dumont, C., Abidi, M. A next-bestview system for autonomous 3-d object reconstruction. Systems, Man and Cybernetics, IEEE Transaction, 30(5), 589-598, 2000.

[16] Amenta, N., Bern, M., and Kamvysselis, M. A new voronoi-based surface reconstruction algorithm. Proceedings on Computer graphics and interactive techniques, SIGGRAPH '98. New York, USA. 415-421, 1998.

[17] Gopi, M. and Krishnan, S. A fast and efficient projectionbased approach for surface reconstruction. Proceeding of Computer Graphics and Image Processing, Brazil, 179-186, 2002.

[18] Sclaroff, S. and Pentland, A. Generalized implicit functions for computer graphics. SIGGRAPH Comput. Graph., 25(4), 247-250, 1991. 